\title{
Toward non-conservative stability conditions for equilibrium points of genetic networks with SUM regulatory functions
}

\author{
Graziano Chesi
}

\begin{abstract}
An important problem in systems biology consists of establishing whether an equilibrium point of a genetic regulatory network is stable. This paper investigates this problem for genetic networks with SUM regulatory functions. It is shown that a sufficient condition for global asymptotical stability of an equilibrium point of these networks can be derived in terms of convex optimizations with LMI constraints by exploiting polynomial Lyapunov functions and SOS techniques. This condition is interesting because does not introduce approximations of the nonlinearities present in the genetic regulatory network, and the conservatism can be decreased by increasing the degree of the involved polynomials.
\end{abstract}

\section{INTRODUCTION}

A primary research area in systems biology is represented by genetic regulatory networks. These networks explain the interactions between genes and proteins to form complex systems that perform complicated biological functions. Genetic regulatory networks are biochemically dynamical systems, and it is natural to model them by using dynamical system models which provide a powerful tool for studying gene regulation processes in living organisms.

Basically, there are two classes of genetic regulatory network models, i.e., the Boolean model (or discrete model) and the differential equation model (or continuous model). In the Boolean models, the activity of each gene is expressed in one of two states, ON or OFF, and the state of a gene is determined by a Boolean function of the states of other related genes. In the differential equation models, the variables describe the concentrations of gene products, such as mRNAs and proteins, as continuous values of the gene regulation systems. See for instance [1]-[3] and references therein.

The class of differential equation models can be divided into subgroups depending on how the concentration of the protein affects the dynamics of the concentration of the mRNA. Among these subgroups, one of interest that has been considered in the literature is represented by genetic networks with SUM regulatory functions. In these genetic regulatory networks, each transcription factor acts additively to regulate a gene, i.e. the regulatory function sums over all the inputs. Consequently, the derivative of the concentration of the mRNA is an affine combination of saturation functions of the concentration of the protein. See for instance [4]-[10] and references therein.

G. Chesi is with the Department of Electrical and Electronic Engineering, University of Hong Kong, Pokfulam Road, Hong Kong, Tel.: +852-22194362, Fax: +852-25598738, Web: http://www.eee.hku.hk/ ${ }^{2}$ chesi
An important problem in genetic regulatory networks consists of establishing whether an equilibrium point is stable or not. In fact, stability expresses the ability of the system to remain in the neighborhood of a certain equilibrium in spite of changes and events which tend to move the state away from it. Unfortunately, establishing whether an equilibrium point is stable or not is a difficult issue, see the references mentioned above and also [11]-[13] which address this problem. Indeed, genetic regulatory networks are nonlinear systems, in particular characterized by combinations of saturation functions, and to determine whether an equilibrium point of such a system is globally asymptotically stable is known to be a NP-hard problem.

This paper investigates stability of equilibrium points of genetic networks with SUM regulatory functions. It is shown that a sufficient condition for global asymptotical stability of an equilibrium point of these networks can be obtained in terms of linear matrix inequality (LMI) feasibility tests, which are convex optimizations [14]. This condition is derived, firstly, by equivalently re-writing these networks as linear systems with static nonlinear constraints, and secondly, by constructing a polynomial Lyapunov function for such a transformed system through the use of techniques based on sum of squares of polynomials (SOS). These techniques are exploited through the Gram matrix method [15], also known as square matricial representation (SMR) [16], which allows one to establish whether a polynomial of arbitrary degree and arbitrary number of variables is SOS via an LMI feasibility test, see for instance [16], [17]. The interest for the proposed stability condition relies on the fact that its conservatism can be decreased by increasing the degree of the polynomials involved in the construction of the optimization. Some numerical examples are reported to illustrate the application and usefulness of the proposed approach.

Before proceeding it is worth mentioning that conditions for investigating stability of an equilibrium point of a genetic regulatory network based on LMIs have been proposed in the literature, in particular [8], [9], [18] (which consider stability in the presence of time-delays, disturbance attenuation, and stability in the presence of uncertainties). The contribution of the proposed approach with respect to these conditions is to provide a different strategy without introducing approximations of the nonlinearities.

The paper is organized as follows. Section II introduces some preliminaries on genetic networks with SUM regulatory functions. Section III describes the proposed results. Section IV presents some illustrative examples. Lastly, Section $\mathrm{V}$ concludes the paper with some final remarks. 


\section{PRELIMINARIES}

\section{A. Problem formulation}

Let us start by introducing the notation adopted throughout the paper:

- $\mathbb{R}$ : real numbers set;

- $\mathbb{R}_{+}:$positive real numbers set, i.e. $\{x \in \mathbb{R}: x \geq 0\}$;

- $0_{n}$ : origin of $\mathbb{R}^{n}$;

- $I_{n}$ : identity matrix $n \times n$;

- $X^{\prime}$ : transpose of matrix $X$;

- $e_{i}: i$ th column of the identity matrix (of size defined by the context);

- $\operatorname{diag}\left(x_{1}, x_{2}, \ldots\right)$ : diagonal matrix with entries $x_{1}, x_{2}, \ldots$ on the diagonal (starting from the topleft corner);

- TF: transcription factor.

In this paper we consider genetic networks with SUM regulatory functions. This class of genetic regulatory networks is described by the model

$$
\left\{\begin{aligned}
\dot{m}_{i}(t) & =-a_{i} m_{i}(t)+\sum_{j=1}^{n} b_{i, j}\left(p_{j}(t)\right) \\
\dot{p}_{i}(t) & =-c_{i} p_{i}(t)+d_{i} m_{i}(t) \\
i & =1, \ldots, n
\end{aligned}\right.
$$

where $m_{i}(t), p_{i}(t) \in \mathbb{R}_{+}$are the concentrations of mRNA and protein of the $i$ th node, and $a_{i}, c_{i}, d_{i} \in \mathbb{R}_{+}$are positive coefficients. The function $b_{i, j}\left(p_{j}(t), 0\right)$ is given by

$$
b_{i, j}\left(p_{j}(t)\right)= \begin{cases}\alpha_{i, j} f\left(p_{j}(t)\right) & \text { if TF } j \text { is an } \\ & \text { activator of gene } i \\ \alpha_{i, j}\left(1-f\left(p_{j}(t)\right)\right) & \text { if } \mathrm{TF} j \text { is a } \\ & \text { repressor of gene } i \\ 0 & \text { otherwise }\end{cases}
$$

where $\alpha_{i, j} \in \mathbb{R}_{+}$are positive coefficients and $f(\cdot)$ is a saturation function, i.e. a function which satisfies

$$
f: \mathbb{R}_{+} \rightarrow[0,1], f(0)=0, f(\infty)=1, f \text { monotonic. }
$$

This function $f(\cdot)$ is typically selected in the class of the Hill's functions, and is hence given by

$$
f\left(p_{i}(t)\right)=\frac{p_{i}(t)^{H}}{\beta^{H}+p_{i}(t)^{H}}
$$

where $\beta \in \mathbb{R}_{+}$and $H$ is a positive integer. Let us define the vectors

$$
m(t)=\left(\begin{array}{c}
m_{1}(t) \\
\vdots \\
m_{n}(t)
\end{array}\right), \quad p(t)=\left(\begin{array}{c}
p_{1}(t) \\
\vdots \\
p_{n}(t)
\end{array}\right) .
$$

Then, the model (1) can be rewritten in matricial form as (see e.g. [8], [9] for details)

$$
\left\{\begin{aligned}
\dot{m}(t) & =-A m(t)+r+R g(p(t)) \\
\dot{p}(t) & =-C p(t)+D m(t)
\end{aligned}\right.
$$

where $A, C, D \in \mathbb{R}_{+}^{n \times n}$ are diagonal matrices with positive components, $R \in \mathbb{R}^{n \times n}$ and $r \in \mathbb{R}_{+}^{n}$ are defined as

$$
R_{i, j}= \begin{cases}\alpha_{i, j} & \text { if } \mathrm{TF} j \text { is an activator of gene } i \\ -\alpha_{i, j} & \text { if } \mathrm{TF} j \text { is a repressor of gene } i \\ 0 & \text { otherwise }\end{cases}
$$

$$
r_{i}=-\sum_{j: R_{i, j}<0} R_{i, j}
$$

and

$$
g(p(t))=\left(f\left(p_{1}(t)\right), \ldots, f\left(p_{n}(t)\right)\right)^{\prime} .
$$

In this paper we address the following problem. Let $\left(m^{*}, p^{*}\right) \in \mathbb{R}_{+}^{2 n}$ be an equilibrium point of the genetic regulatory network (6). Then, the problem consists of establishing whether $\left(m^{*}, p^{*}\right)$ is globally asymptotically stable, i.e.

$$
\lim _{t \rightarrow \infty}\left(\begin{array}{c}
m(t, \bar{m}) \\
p(t, \bar{p})
\end{array}\right)=\left(\begin{array}{c}
m^{*} \\
p^{*}
\end{array}\right) \quad \forall\left(\begin{array}{c}
\bar{m} \\
\bar{p}
\end{array}\right) \in \mathbb{R}_{+}^{2 n}
$$

where $m(t, \bar{m})$ and $p(t, \bar{p})$ denote the solutions at time $t$ of $m(t)$ and $p(t)$ with initial conditions $m(0)=\bar{m}$ and $p(0)=\bar{p}$.

Let us observe that, by solving this problem, we also establish that the equilibrium point is unique since uniqueness is a necessary condition for global asymptotical stability.

In the sequel the dependence on the time $t$ of the considered signals will be omitted for ease of notation unless indicated otherwise.

\section{B. Positive polynomials via SOS Techniques}

Positivity of a polynomial can be investigated via SOS techniques. Specifically, let $p(x)$ be a polynomial of degree $2 m$ in $x \in \mathbb{R}^{n}$, and let $b(x) \in \mathbb{R}^{\sigma(n, m)}$ be a vector containing all monomials of degree less than or equal to $m$ in $x$, being $\sigma(n, m)$ given by

$$
\sigma(n, m)=\frac{(n+m) !}{n ! m !} .
$$

Then, $p(x)$ can be written as

$$
p(x)=b(x)^{\prime} P(\alpha) b(x)
$$

where $P(\alpha) \in \mathbb{R}^{\sigma(n, m) \times \sigma(n, m)}$ is a symmetric affine linear matrix function expressed as

$$
P(\alpha)=P+L(\alpha)
$$

where $P \in \mathbb{R}^{\sigma(n, m) \times \sigma(n, m)}$ is any symmetric matrix such that

$$
p(x)=b(x)^{\prime} P b(x),
$$

$L(\alpha)$ is a linear parametrization of the set

$$
\mathcal{L}=\left\{L=L^{\prime} \in \mathbb{R}^{\sigma(n, m) \times \sigma(n, m)}: b(x)^{\prime} L b(x)=0\right\},
$$

and $\alpha \in \mathbb{R}^{\nu(n, m)}$ is a vector of free parameters, being $\nu(n, m)$ the dimension of $\mathcal{L}$ which is given by

$$
\nu(n, m)=\frac{1}{2} \sigma(n, m)(\sigma(n, m)+1)-\sigma(n, 2 m) .
$$

The representation of $p(x)$ as in (12)-(13) is known as Gram matrix method [15] and SMR [16]. See also [19], [20] where algorithms for the computation of the matrices $P$ and $L(\alpha)$ are reported.

This representation allows one to investigate positivity of polynomials via convex optimizations. Indeed, the condition $p(x)$ is positive, i.e.

$$
p(x) \geq 0 \quad \forall x \in \mathbb{R}^{n},
$$


can be ensured by $p(x)$ is SOS, i.e. there exist polynomials $p_{1}(x), \ldots, p_{k}(x)$ such that

$$
p(x)=\sum_{i=1}^{k} p_{i}(x)^{2}
$$

and this latter condition holds if and only if

$$
\exists \alpha: P(\alpha) \geq 0,
$$

see for instance [16], [17]. The above condition is an LMI feasibility test, which can be solved through a convex optimization, see [14] about LMI feasibility tests. SOS techniques allow one to search for Lyapunov functions, see for instance [17], [21]-[25].

\section{MAIN RESULTS}

In this section we describe the proposed approach, in particular Section III-A derives the sought stability condition and Section III-B investigates its properties.

\section{A. Stability condition}

In this section we describe the proposed approach for investigating global asymptotical stability of an equilibrium point of the genetic regulatory network (6). This approach is derived, firstly, by equivalently re-writing this network as a linear system with static nonlinear constraints, and secondly, by constructing a polynomial Lyapunov function for such a transformed system through the use of the SMR.

Specifically, let us introduce the new variables $x, y \in \mathbb{R}^{n}$

$$
\begin{aligned}
& x=m-m^{*} \\
& y=p-p^{*} .
\end{aligned}
$$

By using these new variables, the system (6) can be rewritten as

$$
\left\{\begin{array}{l}
\dot{x}=-A x+R\left(g\left(y+p^{*}\right)-g\left(p^{*}\right)\right) \\
\dot{y}=-C y+D x
\end{array}\right.
$$

The original equilibrium point $\left(m^{*}, p^{*}\right)$ is hence shifted in the origin. The next step consists of rewriting (21) as a linear system with static nonlinear constraints. To this end, let us define the auxiliary variable $z \in \mathbb{R}^{n}$ as

$$
z=g\left(y+p^{*}\right)-g\left(p^{*}\right) .
$$

It follows that the system (21) can be equivalently described by (22) and

$$
\left\{\begin{array}{l}
\dot{x}=-A x+R z \\
\dot{y}=-C y+D x
\end{array}\right.
$$

From now on, we will focus on the construction of a Lyapunov function candidate proving global asymptotical stability of the origin for the system (22)-(23). Let us indicate with $v(x, y)$ such a Lyapunov function candidate. We select this function in the class of the polynomial functions. Hence, $v(x, y)$ can be written as

$$
v(x, y)=\sum_{\substack{i_{1}+\ldots i_{2 n}=2 \delta_{v} \\ i_{1}, \ldots, i_{2 n} \geq 0}} v_{i_{1}, \ldots, i_{2 n}} x_{1}^{i_{1}} \cdots x_{n}^{i_{n}} y_{1}^{i_{n+1}} \cdots y_{n}^{i_{2 n}}
$$

where $i_{1}, \ldots, i_{2 n}$ are positive integers, $2 \delta_{v}$ is the degree of $v(x, y)$ for some integer $\delta_{v}$, and the quantities $v_{i_{1}, \ldots, i_{2 n}} \in \mathbb{R}$ are the coefficients of $v(x, y)$.

Now, let us consider the temporal derivative $\dot{v}(x, y)$ of the Lyapunov function $v(x, y)$ along the trajectory of the system (22)-(23). To this end, let us define the function

$$
w_{0}(x, y, z)=\nabla v(x, y)\left(\begin{array}{l}
-A x+R z \\
-C y+D x
\end{array}\right) .
$$

We have that

$$
\dot{v}(x, y)=w_{0}(x, y, z) \quad \forall z: \text { (22) holds. }
$$

For each $i=1, \ldots, n$ let us define the function

$$
h_{i}(y, z)=\left(z_{i}+f\left(p_{i}^{*}\right)\right)\left(\beta^{H}+\left(y_{i}+p_{i}^{*}\right)^{H}\right)-\left(y_{i}+p_{i}^{*}\right)^{H} .
$$

We observe that

$$
\begin{gathered}
\text { (22) holds } \\
h_{1}(y, z)=\ldots \stackrel{\mathbb{}}{=} h_{n}(y, z)=0 .
\end{gathered}
$$

Therefore, one has that the temporal derivative $\dot{v}(x, y)$ in (26) can be rewritten as

$$
\dot{v}(x, y)=w_{0}(x, y, z) \quad \forall z: h_{1}(y, z)=\ldots=h_{n}(y, z)=0 .
$$

In order to study $\dot{v}(x, y)$, let us define the function

$$
w_{1}(x, y, z)=w_{0}(x, y, z)+\sum_{i=1}^{n} u_{i}(x, y, z) h_{i}(y, z)
$$

where $u_{i}(x, y, z), 1 \leq i \leq n$, are auxiliary polynomials of some degree $\delta_{u}$. We observe that, for any choice of the polynomials $u_{1}(x, y, z), \ldots, u_{n}(x, y, z)$, one has that

$\dot{v}(x, y)=w_{1}(x, y, z) \quad \forall z: h_{1}(y, z)=\ldots=h_{n}(y, z)=0$.

The next step consists of introducing an appropriate representation of the Lyapunov function candidate $v(x, y)$ and the function $w_{1}(x, y, z)$. By using the SMR introduced in Section II-B, we can express $v(x, y)$ as

$$
v(x, y)=b_{v}(x, y)^{\prime} V b_{v}(x, y)
$$

where $b_{v}(x, y)$ is a vector containing a base for the polynomials in $x$ and $y$ of degree $\delta_{v}$, and $V=V^{\prime}$ is a symmetric matrix containing the coefficients of $v(x, y)$ with respect to $b_{v}(x, y)$. The vector $b_{v}(x, y)$ is chosen under the condition that $b_{v}(x, y)$ vanishes if and only if the pair $(x, y)$ represents the origin, i.e.

$$
\begin{gathered}
\left\|b_{v}(x, y)\right\|=0 \\
\widehat{\mathbb{}} \\
(x, y)=\left(0_{n}, 0_{n}\right) .
\end{gathered}
$$

Similarly we express the function $w_{1}(x, y, z)$. Indeed, let us write the polynomials $u_{1}(x, y, z), \ldots, u_{n}(x, y, z)$ as

$$
u_{i}(x, y, z)=u_{i}^{\prime} b_{u}(x, y, z) \quad \forall i=1, \ldots, n
$$


where $b_{u}(x, y, z)$ is a vector containing a base for the polynomials in $x, y$ and $z$ of degree $\delta_{u}$, and $u_{i}$ is the corresponding vector of coefficients. Let us define the matrix

$$
U=\left(u_{1}, \ldots, u_{n}\right) .
$$

Then, we can rewrite $w_{1}(x, y, z)$ as

$$
w_{1}(x, y, z)=b_{w}(x, y, z)^{\prime}(W(U, V)+L(\alpha)) b_{w}(x, y, z)
$$

where $b_{w}(x, y, z)$ is a vector containing a base for the polynomials in $x, y$ and $z$ of degree $\delta_{w}$ given by

$$
\delta_{w}=\left\lceil\frac{\max \left\{2 \delta_{v}, H+1+\delta_{u}\right\}}{2}\right\rceil .
$$

The vector $b_{w}(x, y, z)$ is chosen under the condition that $b_{w}(x, y, z)$ vanishes if and only if the triplet $(x, y, z)$ represents the origin, i.e.

$$
\begin{gathered}
\left\|b_{w}(x, y, z)\right\|=0 \\
(x, y, z)=\stackrel{\mathbb{1}}{\left(0_{n}, 0_{n}, 0_{n}\right) .}
\end{gathered}
$$

The matrix $W(U, V)$ contains the coefficients of $w_{1}(x, y, z)$ with respect to the chosen $b_{w}(x, y, z)$, and is a symmetric matrix function depending affine linearly on $U$ and $V$. Lastly, $L(\alpha)$ is a linear parametrization of the linear space

$$
\mathcal{L}=\left\{L=L^{\prime}: b_{w}(x, y, z)^{\prime} L b_{w}(x, y, z)=0 \forall x, y, z\right\}
$$

being $\alpha$ a free vector of dimension equal to the dimension of $\mathcal{L}$.

The following theorem shows how the representations (32) and (36) can be exploited to obtain the sought stability condition via convex programming.

Theorem 1: Let $\left(m^{*}, p^{*}\right) \in \mathbb{R}_{+}^{2 n}$ be an equilibrium point of the system (6). Let us suppose that there exist integers $\delta_{v}$ and $\delta_{u}$, matrices $V$ and $U$, and a vector $\alpha$ satisfying the system of LMIs

$$
\left\{\begin{array}{l}
0<V \\
0>W(U, V)+L(\alpha)
\end{array}\right.
$$

Then, $\left(m^{*}, p^{*}\right)$ is globally asymptotically stable.

Proof. Let us suppose that there exist matrices $V$ and $U$ and a vector $\alpha$ such that the system of LMIs (40) holds for some integers $\delta_{v}$ and $\delta_{u}$. From the first inequality of (40) and (32) one has that

$$
\begin{aligned}
& v(x, y)>0 \quad \forall(x, y) \neq 0_{2 n} \\
& v\left(0_{n}, 0_{n}\right)=0 .
\end{aligned}
$$

Then, from the second inequality of (40) and (36) one has that

$$
\begin{aligned}
& w_{1}(x, y, z)<0 \quad \forall(x, y, z) \neq 0_{3 n} \\
& w_{1}\left(0_{n}, 0_{n}, 0_{n}\right)=0 .
\end{aligned}
$$

Let $z$ be any value for which (22) holds. Then, from (28) one has

$$
h_{1}(y, z)=\ldots=h_{n}(y, z)=0,
$$

and hence from (31) it follows that

$$
\begin{aligned}
0 & >w_{1}(x, y, z) \\
& =\dot{v}(x, y)
\end{aligned}
$$

for all $(x, y, z) \neq 0_{3 n}$ with $z$ satisfying (22). This implies that

$$
\dot{v}(x, y)<0 \quad \forall(x, y) \neq\left(0_{n}, 0_{n}\right) .
$$

Therefore, we have that the function $v(x, y)$ is radially unbounded and positive outside the origin, moreover $v(x, y)$ vanishes in the origin, and hence the origin represents the global minimum of $v(x, y)$. In addition, from (45) we have that the temporal derivative $\dot{v}(x, y)$ is negative outside the origin, hence implying that $v(x, y)$ is decreasing along the trajectories of the system (22)-(23), or equivalently the system (6). Therefore, the theorem holds.

Theorem 1 provides a sufficient condition for establishing whether an equilibrium point $\left(m^{*}, p^{*}\right)$ of (6) is globally asymptotically stable in the positive octant. This condition amounts to finding matrices $U$ and $V$ and a vector $\alpha$ such that the inequalities (40) are fulfilled for some integers $\delta_{v}$ and $\delta_{u}$. These inequalities are LMIs, and hence establishing whether (40) holds or not amount to solving an LMI feasibility test, which is a convex optimization as explained, for instance, in [14].

The construction of the matrices $W(U, V)$ and $L(\alpha)$ can be performed by using simple algorithms as those mentioned in Section II-B. The LMI feasibility test (40) can be readily solved by using dedicated software, such as the LMI toolbox [26] or SeDuMi [27].

\section{B. Properties}

First of all, let us observe that the stability condition provided in Theorem 1 differs from existing LMI stability conditions for genetic regulatory networks. In fact, these conditions are based on the approximation of the nonlinearities present in these networks, which are represented by the saturation function $g(p)$ is (6), via sector inclusions as done for instance in [8], [9], [18]. Instead, the condition provided in Theorem 1 does not introduce any approximation of the nonlinearities, and takes into account their exact structure through the polynomials $h_{1}(y, z), \ldots, h_{n}(y, z)$.

Second, let us observe that the conservatism of the stability condition provided in Theorem 1 does not increase as one increases the degrees of the Lyapunov function candidate $v(x, y)$ and auxiliary polynomials $u_{1}(x, y, z), \ldots, u_{n}(x, y, z)$, i.e. the integers $\delta_{v}$ and $\delta_{u}$. Indeed, let us suppose there exist matrices $V$ and $U$ and a vector $\alpha$ satisfying the system of LMIs (40) for some $\delta_{v}$ and $\delta_{u}$. Then, let us define the polynomial

$$
\bar{v}(x, y)=b_{v}(x, y)^{\prime} V b_{v}(x, y)+\hat{v}(x, y)
$$

where $\hat{v}(x, y) \in \mathbb{R}$ is a polynomial composed by monomials of degree greater than or equal to $2 \delta_{v}$, and for $i=1, \ldots, n$ the polynomials

$$
\bar{u}_{i}(x, y, z)=e_{i}^{\prime} U^{\prime} b_{u}(x, y, z)+\hat{u}_{i}(x, y, z)
$$


where $\hat{u}_{i}(x, y, z)$ is a polynomial of degree greater than or equal to $\delta_{u}\left(e_{i}\right.$ is the $i$ th column of the $n \times n$ identity matrix). Let us observe that $\hat{v}(x, y)$ can be chosen such that $\bar{v}(x, y)$ admits a representation analogous to (32) with a positive definite matrix $\bar{V}$ since $V>0$ for assumption. In particular, this can be done by selecting $\hat{v}(x, y)$ of the form

$$
\hat{v}(x, y)=b_{\hat{v}}(x, y)^{\prime} \hat{V} b_{\hat{v}}(x, y)
$$

where $b_{\hat{v}}(x, y)$ is a vector containing a base for the polynomials in $x$ and $y$ with monomials of degree greater than or equal to $\delta_{v}$, and $\hat{V}$ is a positive definite matrix. One has hence:

$$
\bar{v}(x, y)=\left(\begin{array}{l}
b_{v}(x, y) \\
b_{\hat{v}}(x, y)
\end{array}\right)^{\prime} \bar{V}\left(\begin{array}{l}
b_{v}(x, y) \\
b_{\hat{v}}(x, y)
\end{array}\right)
$$

where

$$
\bar{V}=\left(\begin{array}{cc}
V & \\
& \hat{V}
\end{array}\right)
$$

Moreover, $\hat{v}(x, y)$ can be chosen such that its temporal derivative along the trajectory of the system (23) is a SOS polynomial changed in sign. Let us observe, in fact, that (23) depends linearly on the state, and hence $\hat{v}(x, y)$ can be selected as a power of a quadratic function proving stability of (23). This implies that, by simply selecting null polynomials $\hat{u}_{1}(x, y, z), \ldots, \hat{u}_{n}(x, y, z)$, the polynomial $\bar{w}_{1}(x, y, z)$ analogous to $w_{1}(x, y, z)$ admits a representation analogous to (36) with a negative definite matrix $\bar{W}(\bar{U}, \bar{V})+\bar{L}(\bar{\alpha})$, where $\bar{U}$ and $\bar{L}(\bar{\alpha})$ are defined analogously to (35) and (39) respectively. In conclusion, one has hence the property

(40) is feasible for some $\delta_{v}, \delta_{u}$

$$
\Uparrow
$$

(40) is feasible for $\delta_{v}+i, \delta_{u}+j$ for all $i, j \geq 0$.

Lastly, let us consider the necessity of the stability condition provided in Theorem 1. Clearly, since a Lyapunov function proving global asymptotical stability of $\left(m^{*}, p^{*}\right)$ may be non-polynomial in general, it immediately follows that this condition is in general conservative for finite integers $\delta_{v}$ and $\delta_{u}$. Nevertheless, it is interesting to observe that this conservatism can be arbitrary decreased by selecting $\delta_{v}$ and $\delta_{u}$ sufficiently large, since in this way one can approximate non-polynomials to a desired accuracy and cope with the gap existing between positive polynomials and SOS polynomials. Regarding this gap, the reader is referred to [28], [29], [17], [30] and references therein.

\section{ILLUSTRATIVE EXAMPLES}

This section illustrates the proposed approach with some numerical examples. The computational time of the stability condition provided in Theorem 1 is less than 5 seconds for all examples (computational time relative to a Matlab implementation of the proposed condition via SeDuMi [27] on a standard personal computer).

\section{A. Example 1}

Let us consider as first example the repressilator investigated in Escherichia coli [31], which is a genetic network with SUM regulatory functions described by

$$
\left\{\begin{array}{l}
\dot{m}_{i}=-m_{i}+\frac{\gamma_{i}}{1+p_{j}^{2}} \\
\dot{p}_{i}=-\left(p_{i}-m_{i}\right)
\end{array}\right.
$$

where the indexes $i$ and $j$ represents the following genes:

$$
(i, j)=(\text { lacl }, c l),(\text { tet } R, \text { lacl }),(c l, \text { tet } R) .
$$

This genetic regulatory network can be expressed as in (6) with $n=3, H=2, \beta=1$ and

$$
\begin{aligned}
A & =-I_{3} \\
C & =-I_{3} \\
D & =I_{3} \\
R & =\left(\begin{array}{ccc}
0 & 0 & -\gamma_{1} \\
-\gamma_{2} & 0 & 0 \\
0 & -\gamma_{3} & 0
\end{array}\right) \\
r & =\left(\gamma_{1}, \gamma_{2}, \gamma_{3}\right)^{\prime} .
\end{aligned}
$$

We select the plausible values

$$
\gamma_{1}=1, \quad \gamma_{2}=2, \quad \gamma_{3}=5
$$

It follows that this system has an equilibrium point in

$$
\left(m^{*}, p^{*}\right)=(0.27,1.34,1.29,0.27,1.34,1.29)^{\prime} .
$$

The problem consists of establishing whether $\left(m^{*}, p^{*}\right)$ is globally asymptotically stable. To this end, let us use the stability condition provided in Theorem 1 . We hence build the system of LMIs (40) for the simple choice $\delta_{v}=\delta_{u}=1$, and we find out that there exist matrices $U$ and $V$ and a vector $\alpha$ fulfilling these LMIs. Therefore, we conclude that $\left(m^{*}, p^{*}\right)$ is globally asymptotically stable.

For comparison purpose, we attempt to solve the same problem by using existing stability conditions. We find that the conditions proposed in [11] (which is based on the spectral radius of suitable matrices) and [18] (which is based on LMIs via nonlinearities approximation) are not satisfied and do not allow one to conclude global asymptotical stability.

\section{B. Example 2}

As second example let us consider the genetic regulatory network

$$
\left\{\begin{aligned}
\dot{m}_{1}(t) & =-m_{1}(t)+\frac{1}{1+p_{2}^{2}}+\frac{p_{3}^{2}}{1+p_{3}^{2}} \\
\dot{m}_{2}(t) & =-2 m_{2}(t)+\frac{p_{1}^{2}}{1+p_{1}^{2}}+\frac{1}{1+p_{3}^{2}} \\
\dot{m}_{3}(t) & =-3 m_{3}(t)+\frac{1}{1+p_{1}^{2}} \\
\dot{p}_{1}(t) & =-p_{1}(t)+2 m_{1}(t) \\
\dot{p}_{2}(t) & =-0.5 p_{2}(t)+2 m_{2}(t) \\
\dot{p}_{3}(t) & =-p_{3}(t)+m_{3}(t)
\end{aligned}\right.
$$


This genetic regulatory network is characterized by the fact that TF 1 is a repressor of gene 3 and an activator of gene 2 , TF 2 is a repressor of gene 1 , and TF 3 is repressor of gene 2 and an activator of gene 1 .

This genetic regulatory network can be expressed as in (6) with $n=3, H=2, \beta=1$ and

$$
\begin{aligned}
A & =\operatorname{diag}(-1,-2,-3) \\
C & =\operatorname{diag}(-1,-0.5,-1) \\
D & =\operatorname{diag}(2,2,1) \\
R & =\left(\begin{array}{ccc}
0 & -1 & 1 \\
1 & 0 & -1 \\
-1 & 0 & 0
\end{array}\right) \\
r & =(1,1,1)^{\prime} .
\end{aligned}
$$

We have that this genetic regulatory network has an equilibrium point in

$$
\left(m^{*}, p^{*}\right)=(0.24,0.56,0.27,0.47,2.23,0.27)^{\prime} .
$$

The problem consists of establishing whether $\left(m^{*}, p^{*}\right)$ is globally asymptotically stable. We hence use the stability condition provided in Theorem 1, finding that the system of LMIs (40) is feasible for $\delta_{v}=\delta_{u}=1$. This implies that $\left(m^{*}, p^{*}\right)$ is globally asymptotically stable.

\section{CONCLUSiON}

A sufficient condition for global asymptotical stability of an equilibrium point of genetic networks with SUM regulatory functions has been proposed in terms of an LMI feasibility test, which is a convex optimization.

Contrary to existing stability conditions either based on spectral radius or LMI techniques, this stability condition is obtained without introducing approximations of the nonlinearities present in the genetic regulatory network, whose exact structure is taken into account in the optimization. Moreover, the conservatism can be decreased by increasing the degree of the Lyapunov function and other polynomials involved in the optimization.

Future work will investigate the possibility of establishing upper bounds of the degrees of the polynomials required to achieve necessity in the proposed condition.

\section{ACKNOWLEDGMENTS}

The author would like to thank the Associate Editor and the Reviewers for their useful comments.

\section{REFERENCES}

[1] L. Chen and K. Aihara, "Stability of genetic regulatory networks with time delay," IEEE Trans. on Circuits and Systems I, vol. 49, no. 5, pp. 602-608, 2002.

[2] R. Wang, T. Zhou, Z. Jing, and L. Chen, "Modelling periodic oscillation of biological systems with multiple time scale networks," Systems Biology, vol. 1, pp. 71-84, 2004.

[3] C. Li, L. Chen, and K. Aihara, "A systems biology perspective on signal processing in genetic network motifs," IEEE Signal Processing Magazine, vol. 221, no. 3, pp. 136-142, 2007.

[4] C.-H. Yuh, H. Bolouri, and E. H. Davidson, "Genomic cis-regulatory logic: Experimental and computational analysis of a sea urchin gene," Science, vol. 279, pp. 1896-1902, 1998.
[5] P. D'haeseleer, S. Liang, and R. Somogyi, "Gene expression data analysis and modeling," in Pacific Symposium on Biocomputing, Hawaii, USA, 1999.

[6] Y. Setty, A. E. Mayo, M. G. Surette, and U. Alon, "Detailed map of a cis-regulatory input function," in Proc. Nat. Assoc. Sci. USA, vol. 100, 2003, pp. 7702-7707.

[7] S. Kalir, S. Mangan, and U. Alon, "A coherent feed-forward loop with a sum input function prolongs flagella expression in escherichia coli," Molecular Syst. Biol., 2005.

[8] C. Li, L. Chen, and K. Aihara, "Stability of genetic networks with sum regulatory logic: Lure system and LMI approach," IEEE Trans. on Circuits and Systems I, vol. 53, no. 11, pp. 2451-2458, 2006.

[9] — "Stochastic stability of genetic networks with disturbance attenuation," IEEE Transactions on Circuits and Systems II, vol. 54, no. 10, pp. 892-896, 2007.

[10] G. Chesi, "Computing equilibrium points of genetic regulatory networks," Transactions on Computational Systems Biology XI, LNBI 5750, pp. 268-282, 2009.

[11] H. Lu, "On stability of nonlinear continuous-time neural networks with delays," Neural Networks, vol. 13, pp. 1135-1143, 2000.

[12] H. Qi and L. Qi, "Deriving sufficient conditions for global asymptotic stability of delayed neural networks via nonsmooth analysis," IEEE Trans. on Neural Networks, vol. 15, no. 1, pp. 99-109, 2004.

[13] S. Arik and Z. Orman, "Global stability analysis of cohen-grossberg neural networks with time varying delays," Physics Letters A, vol. 341, pp. 410-421, 2005.

[14] S. Boyd, L. El Ghaoui, E. Feron, and V. Balakrishnan, Linear Matrix Inequalities in System and Control Theory. Philadelphia: SIAM, 1994.

[15] M. Choi, T. Lam, and B. Reznick, "Sums of squares of real polynomials," in Symposia in Pure Mathematics, 1995, pp. 103-126.

[16] G. Chesi, A. Tesi, A. Vicino, and R. Genesio, "On convexification of some minimum distance problems," in European Control Conf., Karlsruhe, Germany, 1999.

[17] P. A. Parrilo, "Structured semidefinite programs and semialgebraic geometry methods in robustness and optimization," Ph.D. dissertation, California Institute of Technology, 2000.

[18] G. Chesi and Y. S. Hung, "Stability analysis of uncertain genetic SUM regulatory networks," Automatica, vol. 44, no. 9, pp. 2298-2305, 2008.

[19] G. Chesi, A. Garulli, A. Tesi, and A. Vicino, "Solving quadratic distance problems: an LMI-based approach," IEEE Trans. on Automatic Control, vol. 48, no. 2, pp. 200-212, 2003.

[20] — - Homogeneous Polynomial Forms for Robustness Analysis of Uncertain Systems. Springer, 2009.

[21] A. Papachristodoulou and S. Prajna, "On the construction of Lyapunov functions using the sum of squares decomposition," in IEEE Conf. on Decision and Control, Las Vegas, Nevada, 2002, pp. 3482-3487.

[22] G. Chesi, A. Garulli, A. Tesi, and A. Vicino, "Homogeneous Lyapunov functions for systems with structured uncertainties," Automatica, vol. 39, no. 6, pp. 1027-1035, 2003.

[23] _ _ "Robust analysis of LFR systems through homogeneous polynomial Lyapunov functions," IEEE Trans. on Automatic Control, vol. 49, no. 7, pp. 1211-1216, 2004.

[24] — , "Polynomially parameter-dependent Lyapunov functions for robust stability of polytopic systems: an LMI approach," IEEE Trans. on Automatic Control, vol. 50, no. 3, pp. 365-370, 2005.

[25] —, "Robust stability of time-varying polytopic systems via parameter-dependent homogeneous Lyapunov functions," Automatica, vol. 43, no. 2, pp. 309-316, 2007.

[26] P. Gahinet, A. Nemirovski, A. J. Laub, and M. Chilali, LMI Control Toolbox. The Mathworks Inc., 1995.

[27] J. F. Sturm, "Using SeDuMi 1.02, a MATLAB toolbox for optimization over symmetric cones," Optimization Methods and Software, vol. 1112, pp. 625-653, 1999.

[28] G. Stengle, "A nullstellensatz and a positivstellensatz in semialgebraic geometry," Math. Ann., vol. 207, pp. 87-97, 1974.

[29] B. Reznick, "Some concrete aspects of Hilbert's 17th problem," Contemporary Mathematics, vol. 253, pp. 251-272, 2000.

[30] G. Chesi, "On the gap between positive polynomials and SOS of polynomials," IEEE Trans. on Automatic Control, vol. 52, no. 6, pp. 1066-1072, 2007.

[31] M. B. Elowitz and S. Leibler, "A synthetic oscillatory network of transcriptional regulators," Nature, vol. 403, pp. 335-338, 2000. 